\title{
Determining Ancestry between Rodent- and Human-Derived Virus Sequences in Endemic Foci: Towards a More Integral Molecular Epidemiology of Lassa Fever within West Africa
}

\author{
Ayodeji Olayemi ${ }^{1}$, Adetunji Samuel Adesina ${ }^{2}{ }^{(\mathbb{O}}$, Thomas Strecker ${ }^{3}, \mathrm{~N}^{\prime}$ Faly Magassouba ${ }^{4}$ and \\ Elisabeth Fichet-Calvet ${ }^{5, *}$ \\ 1 Natural History Museum, Obafemi Awolowo University, Ile Ife, HO220005, Nigeria; \\ aolayemi@oauife.edu.ng \\ 2 Department of Biochemistry and Molecular Biology, Obafemi Awolowo University, \\ Ile Ife HO220005, Nigeria; asadesina@yahoo.com \\ 3 Institute of Virology, Philipps University, 35043 Marburg, Germany; strecker@staff.uni-marburg.de \\ 4 Laboratoire des Fièvres Hémorragiques Virales, Conakry, Guinea; cmagassouba01@gmail.com \\ 5 Department of Virology, Bernhard-Nocht Institute of Tropical Medicine, 20359 Hamburg, Germany \\ * Correspondence: fichet-calvet@bnitm.de; Tel.: +49-4042-818-942
}

Received: 6 December 2019; Accepted: 5 February 2020; Published: 7 February 2020

\begin{abstract}
Lassa fever is a viral hemorrhagic illness responsible for thousands of human deaths in West Africa yearly. Rodents are known as natural reservoirs of the causative Lassa mammarenavirus (LASV) while humans are regarded as incidental, spill-over hosts. Analysis of genetic sequences continues to add to our understanding of the evolutionary history, emergence patterns, and the epidemiology of LASV. Hitherto, the source of data in such investigations has mainly comprised human clinical samples. Presently, a rise in the quantity of virus strains accessed through ecological studies over the last 15 years now allows us to explore how LASV sequences obtained from rodents might affect phylogenetic patterns. In this study, we phylogenetically compared LASV sequences obtained from both rodents and humans across West Africa, including those from two localities highly endemic for the disease: Ekpoma in Nigeria and Kenema in Sierra Leone. We performed a time-calibrated phylogeny, using a Bayesian analysis on 198 taxa, including 102 sequences from rodents and 96 from humans. Contrary to expectation, our results show that LASV strains detected in humans within these localities, even those sampled recently, are consistently ancient to those circulating in rodents in the same area. We discuss the possibilities connected to this preliminary outcome. We also propose modalities to guide more comprehensive comparisons of human and rodent data in LASV molecular epidemiological studies.
\end{abstract}

Keywords: Lassa virus; zoonosis; Mastomys; transmission; humans; West Africa; phylogeny; emergence

\section{Introduction}

Lassa fever causes a deadly zoonotic disease that kills 5000 or more people every year in West Africa [1]. The Lassa mammarenavirus (LASV), which causes this illness, is borne by rodents. The Natal multimammate mouse Mastomys natalensis is recognized as the main LASV reservoir while the Guinea multimammate mouse M. erythroleucus and the African wood mouse Hylomyscus pamfi were recently identified as additional hosts [2,3]. Primary (rodent-to-human) transmission of the virus occurs when humans come in contact with excreta or body fluids of infected rodents. Human-to-human spread is regarded as secondary. Further knowledge on the epidemiology of Lassa fever, particularly at the rodent-human interface, is needed to control this disease. 
The recent surge in genetic sequencing has helped to advance our understanding concerning the evolutionary history of LASV. Geographic distribution and estimated emergence dates have been described for four LASV lineages: I-III in Nigeria and IV in Guinea, Sierra Leone, and Liberia [4]. Additional putative lineages were recently detected in southern Mali, northern Ivory Coast, and Togo [5-7].

In addition, a generation of LASV sequences at the local scale during outbreaks within specific countries such as Nigeria and Sierra Leone contributes to the molecular epidemiology of the virus [8-10]. Phylogenetic analyses of these sequences, dominated by clinical samples from humans, commonly depict independent chains of transmission in humans originating from rodents. However, an increasing number of rodent-derived sequences from ecological studies targeted at endemic localities where humans have been regularly sequenced now provide the opportunity to further examine primary LASV transmission [3,11-14].

During outbreaks of rodent-borne viral hemorrhagic fevers, phylogenetic comparison has helped to link infection in human patients to rodent populations for hantaviruses in the USA [15], western Bolivia and Paraguay [16], Finland [17], and Germany [18]. This is also similar for arenaviruses, such as the Lymphocytic choriomeningitis mammarenavirus in France [19] and LASV in southern Mali [6]. These studies typically involved few virus sequences obtained both from the infected patients and rodents in their domestic surroundings, and do not allow a statistical comparison between rodent versus human hosts.

Here, we seek to phylogenetically infer ancestry and descent between LASV sequences detected in rodents and humans in selected localities within West Africa in order to provide increased insight into virus transmission at the rodent-human boundary. Our aim was to compile a dataset including LASV sequences collected from both humans and Mastomys rodents within distinctive hotspots of Lassa fever. Two localities in West Africa currently fit this description: Kenema (represented by Kenema and other closely surrounding localities within a $35 \mathrm{~km}$ radius, Sierra Leone) and Ekpoma (comprising Ekpoma and Eguare Egoro, only $6 \mathrm{~km}$ from each other within Nigeria).

\section{Materials and Methods}

\subsection{LASV Sequences in Mastomys}

We used complete LASV sequences from Mastomys spp. trapped in Guinea $(n=5)$, Mali $(n=4)$, Nigeria $(n=2)$, and Sierra Leone $(n=10)[3,6,8,20,21]$. Since these full genomes are few in number, we supplemented them with partial sequences from other Mastomys trapped in Guinea and Nigeria. In Guinea, we sequenced the GP $1 \mathrm{~kb}$ portion to complement NP sequences published in Lecompte et al. [12] and Fichet-Calvet et al. [11]. A fragment of 950 nucleotides was amplified using a RT-PCR protocol with the following primers: LVS 36-fwd (ACCGGGGATCCTAGGCATTT) and OWS 1000-rev (AGCATGTCACAGAAYTCYTCATCATG) for the strains from Faranah and Denguedou. In Nigeria, we sequenced the NP to complement LASV GP sequences published in Olayemi et al. [14] from Mastomys natalensis that were sampled during 2011-2012. We designed primers to amplify the NP at the same position of those published in Bowen et al. [4]. A fragment of 800 nucleotides was amplified using a RT-PCR protocol (supplementary information) with the following primers: LVSnig 1669-fwd (TATATTGAGTCCTCCTGACACAG) and LVSnig 2511-rev (TGTTGGAGACCATCAAGGTT) (Table S1). Furthermore, to expand the timeframe of our collection in Ekpoma, we added LASV GP $1 \mathrm{~kb}$ and partial NP sequences from 13 M. natalensis trapped during 2014-2016. All PCR fragments were sequenced on both strands. The sequences were assembled and aligned using the Mac Vector software (Mac Vector 16.0.8, Inc, Apex North Carolina 27502, USA). In total, we generated 46 partial GP sequences of LASV from M. natalensis in Faranah and in Denguedou; 13 partial NP from M. natalensis in Ekpoma collected in 2011-2012; and GP 1kb plus partial NP from 13 M. natalensis also trapped in Ekpoma during 2014-2016 (Table S2). Sequences were submitted to GenBank under accession numbers 
MN123651-MN123709 and MT001745-MT001770. The sequences from Mastomys used in our study are listed in Table S2.

\subsection{LASV Sequences in Humans}

The sequences of LASV isolated in humans are readily available in Genbank, thanks in particular to the research of Andersen et al. [8] and Siddle et al. [10]. They mainly concern lineages II and IV circulating around Ekpoma in Nigeria and Kenema in Sierra Leone, respectively. In this article, we use the new Nigeria sequences published in 2018 because they are localized by state. For Sierra Leone, however, we use those of 2015 [8] differentiating sequences from Kenema, Bo, and Liberia. The sequences from humans used in our study are listed in Table S3.

\subsection{Phylogenetic Analysis}

The phylogenies were inferred by the Bayesian Markov Chain Monte Carlo (MCMC) method implemented in BEAST software (https://beast.community) [22].

Model 1: To evaluate the sequences from humans living near Kenema only, we did a preliminary analysis gathering all the known LASV strains, including those from known areas. In BEAUTI, the parameters are:

- One partition, NP full sequence for 226 taxa;

- Substitution model as GTR + gamma without codon partition;

- Lognormal relax clock;

- Coalescent tree with a constant size population;

- $\mathrm{MCMC}=10 \mathrm{M}$, echo states, and log parameters every 10,000.

It was therefore possible to remove the samples coming from Bo (G1442, G1529, G2141, G2230, G2259, G2363, G2431, G2557, G2615, G2723, G2789, G3034, G3278) and those from Liberia from the Kenema dataset. These samples correspond to patients tested in Kenema but coming from neighboring areas.

Model 2: To get an estimation of the time of divergence, with both humans and Mastomys, we merged the GP and NP in a combined phylogenetic analysis. As we were searching for LASV diversity, all similar sequences were removed.

In BEAUTI, the parameters are:

- Two partitions, GP $900 \mathrm{nt}$ and NP $712 \mathrm{nt}$ for 198 sequences. The substitution models, clock, and trees are linked;

- Eight taxa were defined: Homo Ekpoma, Homo Kenema, Mastomys Denguedou, Mastomys Ekpoma, Mastomys Faranah, Mastomys Kenema, Mastomys Madina, and Mastomys Mali;

- Tip dates at the nearest day;

- Substitution model as GTR + gamma and codon partition with positions 1,2,3;

- $\quad$ Strict (model 2a) or uncorrelated relaxed (model 2b) or random local (model 2c) or fixed local (model 2d) clock;

- Coalescent tree with a constant size population;

- $\mathrm{MCMC}=50 \mathrm{M}$, echo states, and log parameters every 50,000.

The $\mathrm{xml}$ files issued from BEAUTI were run in BEAST, the log files checked in TRACER, and consensus trees were visualized through FigTree (BEAST packages, https://beast.community/programs).

\section{Results}

\subsection{LASV in Mastomys}

The six viral populations isolated from $M$. natalensis and M. erythroleucus show very different emergence periods according to the rodent populations from which they originated in West Africa 
(Figure 1a, Table 1). Indeed, the LASV clade obtained from rodents in Mali is the oldest and dates back to 96 years, whereas that obtained in Denguedou near the Sierra Leonean border is very recent and seems to have emerged 20 years ago. Between these two emerged the LASV population of Faranah in Upper Guinea; those from Ekpoma and Kenema synchronously; and then that of Madina Oula in coastal Guinea (Figure 1b). The pattern is similar regardless of clock model; strict or uncorrelated lognormal (Table 1). We did not present the results of the random and fixed local clock because the models never converged well, i.e., the ESS parameters did not reach the value of 100 after a MCMC chain of $50 \mathrm{M}$. Figure $1 \mathrm{c}$ shows the phylogenetic tree gathering the rodent- and human-derived LASV sequences in West Africa.

Table 1. Times of the most recent common ancestor (tmrca) in years according to each model; $2 \mathrm{a}=$ strict clock, $2 \mathrm{~b}=$ uncorrelated relaxed clock.

\begin{tabular}{ccc}
\hline Taxon & Model 2a & Model 2b \\
\hline Homo Ekpoma & $65(54-76)$ & $63(50-77)$ \\
Homo Kenema & $66(58-76)$ & $66(55-77)$ \\
Mastomys Denguedou & $20(18-23)$ & $20(17-24)$ \\
Mastomys Ekpoma & $56(47-64)$ & $54(45-64)$ \\
Mastomys Faranah & $75(64-87)$ & $71(59-85)$ \\
Mastomys Kenema & $54(49-59)$ & $53(49-59)$ \\
Mastomys Madina & $39(32-46)$ & $38(29-47)$ \\
Mastomys Mali & $96(81-113)$ & $89(69-112)$ \\
\hline
\end{tabular}

\subsection{LASV in Homo}

To compare the sequences obtained from humans to those from rodents, we have only two sites with sufficient numbers of individual human-derived sequences for analysis: Ekpoma in Nigeria and Kenema in Sierra Leone. This analysis is summarized in Figure 2 and Table 1 where we note the same pattern: the sequences observed in Homo are on average earlier than those detected in Mastomys. A superimposition exists between the two viral populations, which indicates a close contact and the passage of the virus from one host to another. On the Kenema graph (Figure 2b), the two peaks are more disjointed than in Ekpoma (Figure 2a), probably because of a greater distance between the sites sampled within this locality. Additional analyses that do not take into account cases from Bo or Liberia show even more disjointed peaks (data not shown).

\section{Discussion}

\subsection{LASV Emergence Per Locality}

In the absence of the complete S segment, a combination of partial GP and NP sequences to increase genome information offers a good compromise (1612 nt together in one analysis instead of two separate analyses with GP, $900 \mathrm{nt}$, and NP, $712 \mathrm{nt}$, respectively). This combination with two partial genes in the open reading frame has never been done before. However, our analysis showed more recent dates of divergence compared to studies done on the complete $\mathrm{S}$ segment. For instance, the date of divergence in Sierra Leone is 107 (91-124) years in our analysis versus 118 (102-137) in the Andersen study [8]. Divergence out of Nigeria is 249 (212-284) years in our study versus 283 (241-331) described by Andersen et al. We therefore suggest that the dates presented in this analysis are slightly underestimated because we used a shorter nucleotide sequence than the complete $\mathrm{S}$ segment (1612 nt instead of $\pm 3400 \mathrm{nt}$ ). As we consider that the bias is similar for each strain, the comparison between the different viral populations remains valuable. 


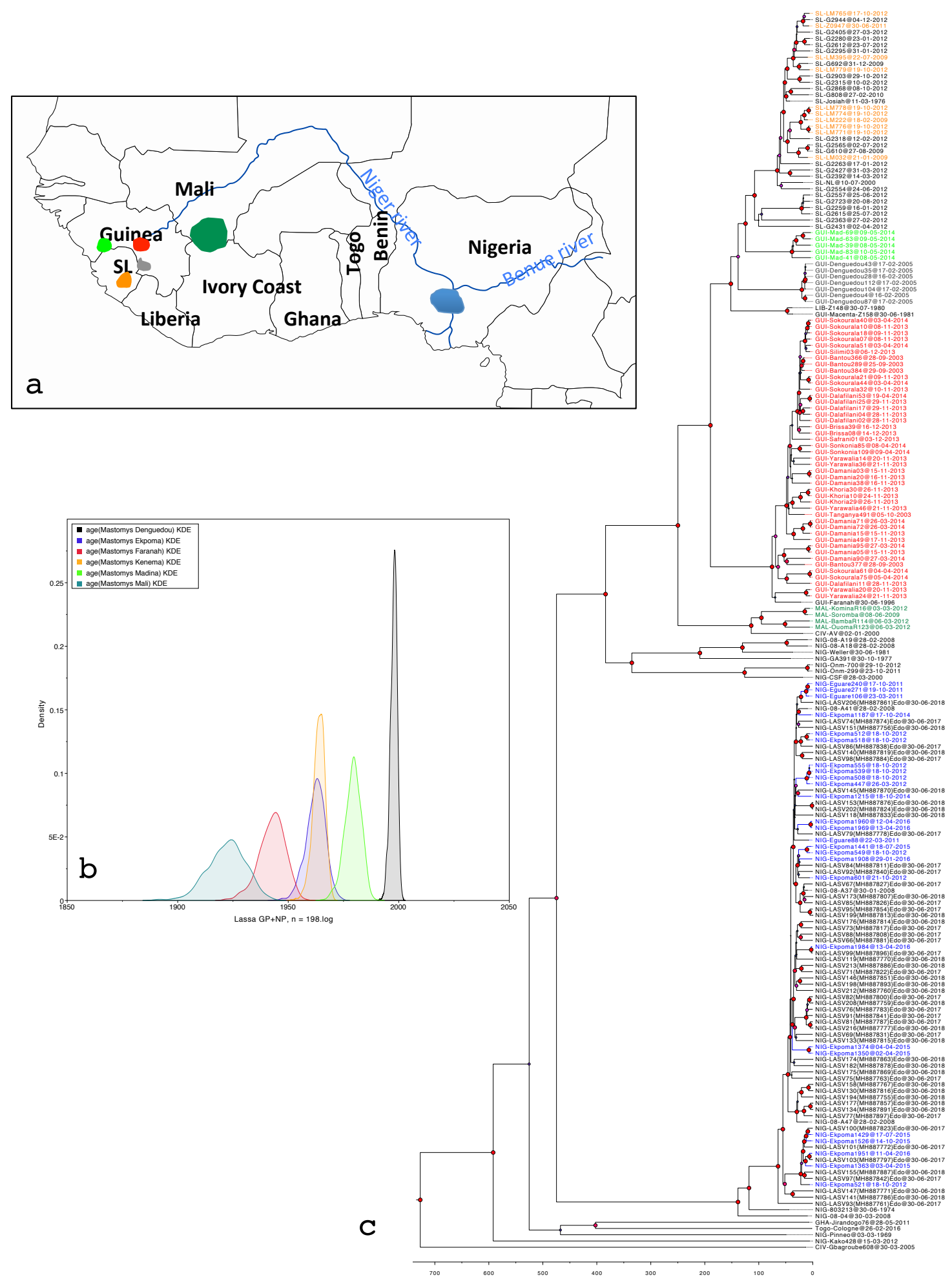

Figure 1. (a) Map of West Africa showing the geographic distribution of Lassa mammarenavirus (LASV) in Mastomys populations represented in the phylogenetic tree in the same colors. (b) LASV dates of emergence among these Mastomys populations (X axis). Density on the $\mathrm{Y}$ axis represents the probability of clock rate validity. (c) Phylogenetic tree featuring LASV sequences derived from humans (in black); from Mastomys natalensis in Ekpoma, Nigeria (blue); southern Mali (dark green); Kenema, Sierra Leone (orange); Denguedou, Forest Guinea (gray); Faranah, Upper Guinea (red); and from $M$. erythroleucus in Madina Oula, Guinea (light green). The tree combines GP and NP sequences analyzed in model 2a (strict clock, see Table 1). Red dots at the nodes indicate a posterior value of $>0.9$. 

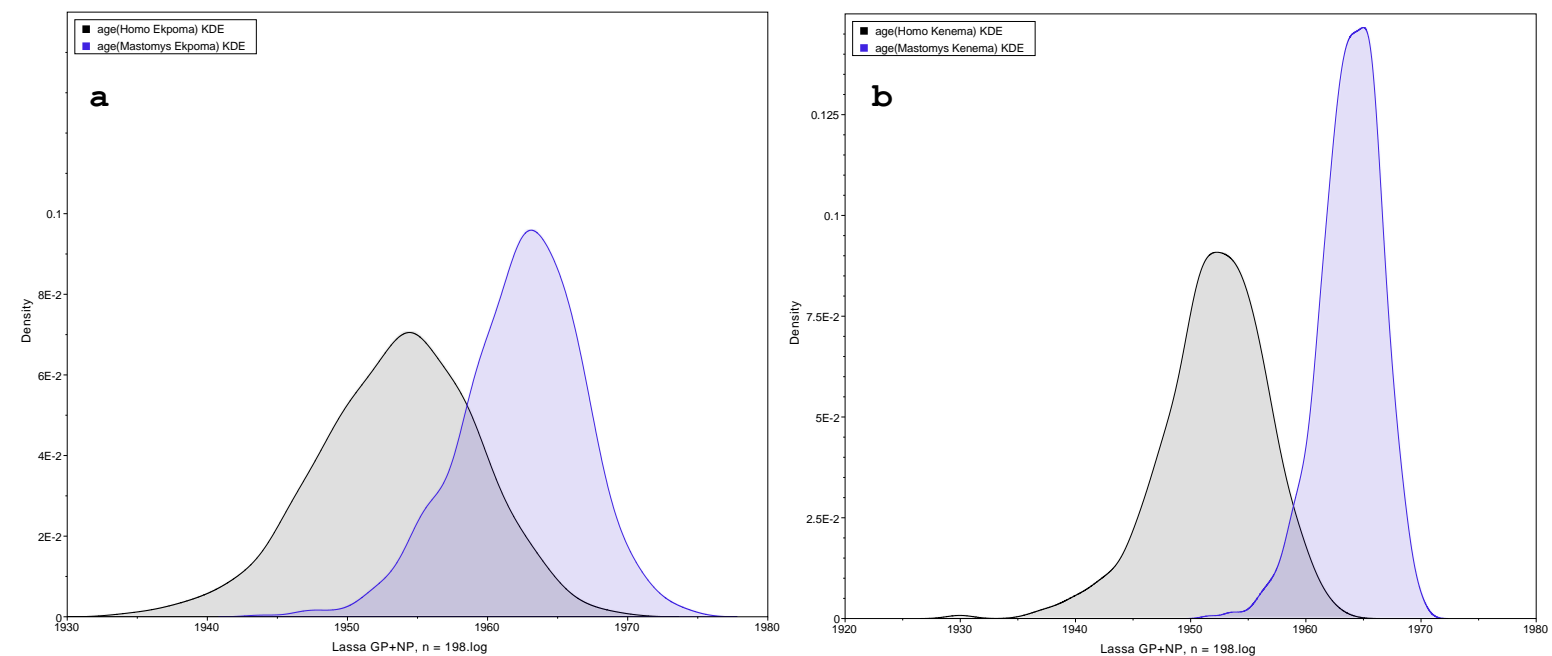

Figure 2. Dates of LASV emergence in Mastomys natalensis versus Homo sapiens living in (a) Ekpoma (Nigeria) and (b) Kenema (Sierra Leone). In both locations, the peak in humans shows the emergence of LASV earlier than the one in rodents. Peaks in humans include 58 sequences in Nigeria and 19 sequences in Sierra Leone. Peaks in rodents include 26 sequences in Nigeria and 10 in Sierra Leone (see Tables S2 and S3).

The viral population from Malian rodents is the oldest (96 years), and we can suggest that the virus was then exported to Faranah, South Upper Guinea, 20 years later, and lately to Sierra Leone with another jump of 20 years. The viral population found in Denguedou, a locality between the Sierra Leonean/Guinean border and Guekedou, is probably a signature of human displacements during the civil war in Sierra Leone (1994-2004) as it emerged only 20 years ago. During that time, 700,000 Sierra Leonean refugees arrived in Forest Guinea and were dispatched all along the border. The times of the most recent common ancestor (tmrca) estimated in our study is higher than the one estimated in Lalis et al. [23] because we used the GP in addition to a short NP fragment. The recent viral population discovered in Madina Oula in M. erythroleucus [3] emerged between those observed in Denguedou and those observed in Kenema. These sequences are phylogenetically very different as they cluster to Liberian strains. The village of Madina Oula regularly receives some loggers from Forest Guinea who probably imported this strain. Finally, the strains found in the Nigerian Mastomys from Ekpoma are quite recent, \pm 55 years, similarly to those found in the Kenema area.

\subsection{LASV Emergence Per Host}

Phylogenetic clustering in the clades representing Kenema (within lineage IV) and Ekpoma (in lineage II) show that human-derived LASV sequences are significantly ancestral to those obtained from the M. natalensis rodent, regardless of time of sampling. Normally, one would have expected rodent-to-human transmission (where long-term evolution of the virus takes place in rodents and spills over intermittently to humans) to be consistent with the LASV strains from M. natalensis being generally ancestral or at least randomly interspersed on the phylogenetic tree compared to those obtained from humans [13]. Therefore, our results may suggest the occurrence of human-to-rodent transmission (reverse zoonosis).

During epidemics of certain diseases, patterns of ancestry and descent have helped determine that virus routes of transmission were from humans to other mammals. For instance, investigations in Sri Lanka and Mexico concerning Influenza A (H1N1 and H3N2) emergence in a cluster of farms showed that virus transmission between humans preceded infection in swine [24,25]. Other diseases such as rabies, hepatitis E, and respiratory syndromes were recently proved to be transmitted by humans to dogs, raw pork, and chimpanzees [26-29]. The idea that humans may function in some circumstances as "reservoir hosts" of LASV is counter-intuitive, as infection is usually associated with acuteness, 
severe illness and mortality. However, there are also data demonstrating that up to $70 \%$ of LASV seropositive humans show benign or no symptoms [1]. Moreover, those recovered from the disease may continue to shed the virus in urine or saliva for up to 60 days [1,30], contaminating domestic surfaces, waste, and sewage systems accessible to rodents.

We are aware that the notion of reverse zoonosis in Lassa fever would require a large body of additional evidence to become established, including experimental approaches. Furthermore, as we envisage the impending availability of a much expanded pool of rodent-sourced LASV sequences against which our findings can be tested, we regard our research as a precursory, yet important foundation that will inform subsequent data collection and analyses.

\subsection{Future Sampling}

Appropriateness and robustness in data acquisition lend to interpretability. In Ekpoma, for instance, which is known as one of the most endemic localities for Lassa fever in Nigeria [31], we conducted rodent trapping in 24 GPS-tracked sites spanning the city, including precise addresses where Lassa fever cases were previously recorded. We sampled these sites twice each year during 2011-2012 (Olayemi et al. [14]) and in eight sessions through 2014-2016. Therefore, we find it surprising that, considering the geographic and temporal scope of our compilation, almost all the LASV strains we sequenced from M. natalensis were phylogenetically younger in comparison to those obtained from humans.

In addition to the structured sampling in the ecological projects that generated most of the rodent-derived LASV sequences, we suggest conducting opportunistic collection approaches in future studies. In such a case, Mastomys would be captured in the homes of Lassa fever patients as soon as they are diagnosed. This would result in a greater probability of detecting the older strains that have already been documented in humans; if they are indeed circulating in rodents. Studies in molecular epidemiology such as ours can be notoriously confounded by misleading human geographic data (due to movement after infection or poor hospital records [13]), causing virus strains from out-of-town to be labeled for a certain locality; and, in turn, influencing the interpretation of ancestry and descent for strains in that locality.

Thus, future studies should aim to link human and rodent data more directly at a finer scale (i.e., at the level of the home address), producing multiple, comparable phylogenetic reconstructions of who-infected-who within a locality. This will help to further support our findings and will provide much needed insight underlying LASV transmission mechanisms at the rodent-human interface.

\section{Conclusions}

Our findings do not necessarily contradict the rodent-to-human route of LASV transmission, which is supported by recent LASV phylogenies based on sequences mostly sampled from humans [8-10]. Instead, we call for a broader paradigm including infection in the reverse direction. In such a scenario, few infectious humans could initiate a LASV focus area, which is then perpetuated by commensal rodents acting as transmission enhancers.

Supplementary Materials: The following are available online at http://www.mdpi.com/2079-7737/9/2/26/s1, Table S1: RT-PCR protocol targeted on Nigerian LASV nucleoprotein, Table S2: List of LASV sequences from rodents used in the analysis $(n=102)$, Table S3: List of LASV sequences from humans used in the analysis $(n=96)$.

Author Contributions: Conceptualization, methodology, and formal analysis, E.F.-C.; investigation, A.O., A.S.A., T.S., N.M., and E.F.-C.; writing-original draft preparation, A.O., and E.F.-C.; writing-review and editing, A.O., A.S.A., T.S., N.M., and E.F.-C. All authors have read and agreed to the published version of the manuscript.

Funding: This research was funded by the European Foundation Initiative for African Research into Neglected Tropical diseases (EFINTD), grant numbers 1/85/022 and 89 540, and the German Research Foundation (DFG), grant numbers FI 1781/2-1, GU 883/4-1, and 197785619/SFB1021.

Acknowledgments: We are grateful to Lina Moses for providing accurate dates and locations of rodent captures in Sierra Leone. 
Conflicts of Interest: The authors declare no conflict of interest. The funders had no role in the design of the study; in the collection, analyses, or interpretation of data; in the writing of the manuscript, or in the decision to publish the results.

\section{References}

1. McCormick, J.B.; Fisher-Hoch, S.P. Lassa fever. Curr. Top Microbiol. Immunol. 2002, 262, 75-109. [PubMed]

2. Monath, T.P.; Newhouse, V.F.; Kemp, G.E.; Setzer, H.W.; Cacciapuoti, A. Lassa virus isolation from Mastomys natalensis rodents during an epidemic in Sierra Leone. Science 1974, 185, 263-265. [CrossRef] [PubMed]

3. Olayemi, A.; Cadar, D.; Magassouba, N.; Obadare, A.; Kourouma, F.; Oyeyiola, A.; Fasogbon, S.; Igbokwe, J.; Rieger, T.; Bockholt, S.; et al. New Hosts of The Lassa Virus. Sci. Rep. 2016, 6, 25280. [CrossRef] [PubMed]

4. Bowen, M.D.; Rollin, P.E.; Ksiazek, T.G.; Hustad, H.L.; Bausch, D.G.; Demby, A.H.; Bajani, M.D.; Peters, C.J.; Nichol, S.T. Genetic diversity among Lassa virus strains. J. Virol. 2000, 74, 6992-7004. [CrossRef]

5. Kouadio, L.; Nowak, K.; Akoua-Koffi, C.; Weiss, S.; Allali, B.K.; Witkowski, P.T.; Kruger, D.H.; Couacy-Hyman, E.; Calvignac-Spencer, S.; Leendertz, F.H. Lassa Virus in Multimammate Rats, Cote d'Ivoire 2013. Emerg. Infect. Dis. 2015, 21, 1481-1483. [CrossRef]

6. Safronetz, D.; Lopez, J.E.; Sogoba, N.; Malga, O.; Dahlstrom, E.; Zivcec, M.; Feldmann, F.; Haddock, E.; Fischer, R.J.; Anderson, J.M.; et al. Detection of Lassa virus, Mali. Emerg. Infect. Dis. 2010, 16, 1123-1126. [CrossRef]

7. Whitmer, S.L.M.; Strecker, T.; Cadar, D.; Dienes, H.P.; Faber, K.; Patel, K.; Brown, S.M.; Davis, W.G.; Klena, J.D.; Rollin, P.E.; et al. New Lineage of Lassa Virus, Togo, 2016. Emerg. Infect. Dis. 2018, 24, 599-602. [CrossRef]

8. Andersen, K.G.; Shapiro, B.J.; Matranga, C.B.; Sealfon, R.; Lin, A.E.; Moses, L.M.; Folarin, O.A.; Goba, A.; Odia, I.; Ehiane, P.E.; et al. Clinical Sequencing Uncovers Origins and Evolution of Lassa Virus. Cell 2015, 162, 738-750. [CrossRef]

9. Kafetzopoulou, L.E.; Pullan, S.T.; Lemey, P.; Suchard, M.A.; Ehichioya, D.U.; Pahlmann, M.; Thielebein, A.; Hinzmann, J.; Oestereich, L.; Wozniak, D.M.; et al. Metagenomic sequencing at the epicenter of the Nigeria 2018 Lassa fever outbreak. Science 2019, 363, 74-77. [CrossRef]

10. Siddle, K.J.; Eromon, P.; Barnes, K.G.; Mehta, S.; Oguzie, J.U.; Odia, I.; Schaffner, S.F.; Winnicki, S.M.; Shah, R.R.; Qu, J.; et al. Genomic Analysis of Lassa Virus during an Increase in Cases in Nigeria in 2018. N. Engl. J. Med. 2018, 379, 1745-1753. [CrossRef]

11. Fichet-Calvet, E.; Olschlager, S.; Strecker, T.; Koivogui, L.; Becker-Ziaja, B.; Camara, A.B.; Soropogui, B.; Magassouba, N.; Gunther, S. Spatial and temporal evolution of Lassa virus in the natural host population in Upper Guinea. Sci. Rep. 2016, 6, 21977. [CrossRef] [PubMed]

12. Lecompte, E.; Fichet-Calvet, E.; Daffis, S.; Koulémou, K.; Sylla, S.; Kourouma, F.; Doré, A.; Soropogui, B.; Aniskin, V.; Allali, B.; et al. Mastomys natalensis and Lassa fever, West Africa. Emerg. Infect. Dis. 2006, 12, 1971-1974. [CrossRef] [PubMed]

13. Leski, T.A.; Stockelman, M.G.; Moses, L.M.; Park, M.; Stenger, D.A.; Ansumana, R.; Bausch, D.G.; Lin, B. Sequence variability and geographic distribution of Lassa virus, Sierra Leone. Emerg. Infect. Dis. 2015, 21,609-618. [CrossRef] [PubMed]

14. Olayemi, A.; Obadare, A.; Oyeyiola, A.; Igbokwe, J.; Fasogbon, S.; Igbahenah, F.; Ortsega, D.; Asogun, D.; Umeh, P.; Vakkai, I.; et al. Arenavirus Diversity and Phylogeography of Mastomys natalensis Rodents, Nigeria. Emerg. Infect. Dis. 2016, 22, 687-690. [CrossRef] [PubMed]

15. Hjelle., B.; Torrez-Martinez, N.; Koster, F.T.; Jay, M.; Ascher, M.S.; Brown, T.; Reynolds, P.; Ettestad, P.; Voorhees, R.E.; Sarisky, J.; et al. Epidemiologic linkage of rodent and human hantavirus genomic sequences in case investigations of hantavirus pulmonary syndrome. J. Infect. Dis. 1996, 173, 781-786. [CrossRef] [PubMed]

16. Johnson, A.M.; Bowen, M.D.; Ksiazek, T.G.; Johnson, A.M.; Bowen, M.D.; Ksiazek, T.G.; Williams, R.J.; Bryan, R.T.; Mills, J.N.; Peters, C.J.; et al. Laguna Negra virus associated with HPS in western Paraguay and Bolivia. Virology 1997, 238, 115-127. [CrossRef] [PubMed]

17. Plyusnin, A.; Mustonen, J.; Asikainen, K.; Plyusnina, A.; Niemimaa, J.; Henttonen, H.; Vaheri, A. Analysis of puumala hantavirus genome in patients with nephropathia epidemica and rodent carriers from the sites of infection. J. Med. Virol. 1999, 59, 397-405. [CrossRef] 
18. Schilling, S.; Emmerich, P.; Klempa, B.; Auste, B.; Schnaith, E.; Schmitz, H.; Kruger, D.H.; Gunther, S.; Meisel, H. Hantavirus disease outbreak in Germany: Limitations of routine serological diagnostics and clustering of virus sequences of human and rodent origin. J. Clin. Microbiol. 2007, 45, 3008-3014. [CrossRef]

19. Emonet, S.; Retornaz, K.; Gonzalez, J.P.; de Lamballerie, X.; Charrel, R.N. Mouse-to-human transmission of variant lymphocytic choriomeningitis virus. Emerg. Infect. Dis. 2007, 13, 472-475. [CrossRef]

20. Ehichioya, D.U.; Hass, M.; Becker-Ziaja, B.; Ehimuan, J.; Asogun, D.A.; Fichet-Calvet, E.; Kleinsteuber, K.; Lelke, M.; Meulen, J.; Akpede, G.; et al. Current molecular epidemiology of Lassa virus in Nigeria. J. Clin. Microbiol. 2011, 49, 1157-1161. [CrossRef]

21. Safronetz, D.; Sogoba, N.; Lopez, J.E.; Maiga, O.; Dahlstrom, E.; Zivcec, M.; Feldmann, F.; Haddock, E.; Fischer, R.J.; Anderson, J.M.; et al. Geographic distribution and genetic characterization of Lassa virus in sub-Saharan Mali. PLoS Negl. Trop. Dis. 2013, 7, e2582. [CrossRef]

22. Drummond, A.J.; Suchard, M.A.; Xie, D.; Rambaut, A. Bayesian phylogenetics with BEAUti and the BEAST 1.7. Mol. Biol. Evol. 2012, 29, 1969-1973. [CrossRef] [PubMed]

23. Lalis, A.; Leblois, R.; Lecompte, E.; Denys, C.; Ter Meulen, J.; Wirth, T. The impact of human conflict on the genetics of Mastomys natalensis and Lassa virus in West Africa. PLoS ONE 2012, 7, e37068. [CrossRef] [PubMed]

24. Nelson, M.I.; Souza, C.K.; Trovao, N.S. Human-Origin Influenza A(H3N2) Reassortant Viruses in Swine, Southeast Mexico. Emerg. Infect. Dis. 2019, 25, 691-700. [CrossRef] [PubMed]

25. Perera, H.K.; Vijaykrishna, D.; Premarathna, A.G.; Jayamaha, C.J.S.; Wickramasinghe, G.; Cheung, C.L.; Yeung, M.F.; Poon, L.L.M.; Perera, A.K.C.; Barr, I.G.; et al. Molecular epidemiology of influenza A(H1N1)pdm09 virus among humans and swine, Sri Lanka. Emerg. Infect. Dis. 2014, 20, 2080-2084. [CrossRef]

26. Geng, Y.; Zhao, C.; Guo, T.; Xu, Y.; Wang, W.; Liu, H.; Wang, Y. Detection of Hepatitis E Virus in Raw Pork and Pig Viscera As Food in Hebei Province of China. Foodborne Pathog. Dis. 2019, 16, 325-330. [CrossRef]

27. Negrey, J.D.; Reddy, R.B.; Scully, E.J.; Phillips-Garcia, S.; Owens, L.A.; Langergraber, K.E.; Mitani, J.C.; Emery, T.M.; Wrangham, R.W.; Muller, M.N.; et al. Simultaneous outbreaks of respiratory disease in wild chimpanzees caused by distinct viruses of human origin. Emerg. Microbes Infect. 2019, 8, 139-149. [CrossRef]

28. Olarinmoye, A.O.; Kamara, V.; Jomah, N.D.; Olusaga, B.O.; Ishola, O.O.; Kamara, A.; Luka, P.D. Molecular detection of rabies virus strain with $\mathrm{N}$-gene that clustered with China lineage 2 co-circulating with Africa lineages in Monrovia, Liberia: First reported case in Africa. Epidemiol. Infect. 2019, 147, e85. [CrossRef]

29. Nelson, M.I.; Vincent, A.L. Reverse zoonosis of influenza to swine: New perspectives on the human-animal interface. Trends Microbiol. 2015, 23, 142-153. [CrossRef]

30. Lunkenheimer, K.; Hufert, F.T.; Schmitz, H. Detection of Lassa virus RNA in specimens from patients with Lassa fever by using the polymerase chain reaction. J. Clin. Microbiol. 1990, 28, 2689-2692. [CrossRef]

31. Asogun, D.A.; Adomeh, D.I.; Ehimuan, J.; Odia, I.; Hass, M.; Gabriel, M.; Olschlager, S.; Backer-Ziaja, B.; Folarin, O.; Phelan, E.; et al. Molecular diagnostics for Lassa fever at Irrua Specialist Teaching Hospital, Nigeria: Lessons learnt from two years of laboratory operation. PLoS Negl. Trop. Dis. 2012, 6, e1839. [CrossRef] [PubMed]

(C) 2020 by the authors. Licensee MDPI, Basel, Switzerland. This article is an open access article distributed under the terms and conditions of the Creative Commons Attribution (CC BY) license (http://creativecommons.org/licenses/by/4.0/). 